\title{
ON $S$-CURVATURE OF A HOMOGENEOUS FINSLER SPACE WITH RANDERS CHANGED SQUARE METRIC *
}

\author{
Sarita Rani and Gauree Shanker
}

(C) by University of Niš, Serbia | Creative Commons Licence: CC BY-NC-ND Abstract. The study of curvature properties of homogeneous Finsler spaces with $(\alpha, \beta)$ metrics is one of the central problems in Riemann-Finsler geometry. In the present paper, the existence of invariant vector fields on a homogeneous Finsler space with Randers changed square metric has been proved. Further, an explicit formula for $S$ curvature of Randers changed square metric has been established. Finally, using the formula of $S$-curvature, the mean Berwald curvature of afore said $(\alpha, \beta)$-metric has been calculated.

Keywords: Homogeneous Finsler space, square metric, Randers change, invariant vector field, S-curvature, mean Berwald curvature.

\section{Introduction}

According to S. S. Chern [6], Finsler geometry is just Riemannian geometry without quadratic restriction. Finsler geometry is an interesting and active area of research for both pure and applied reasons $[2,1,13,16]$. In 1972, M. Matsumoto [17] introduced the concept of $(\alpha, \beta)$-metrics which are the generalizations of Randers metric introduced by G. Randers [20]. Z. Shen [25] introduced the notion of $S$-curvature, a non-Riemannian quantity, for a comparison theorem in Finsler geometry. It is non-Riemannian in the sense that any Riemannian manifold has vanishing $S$-curvature. One special class of Finsler spaces is homogeneous and symmetric Finsler spaces. It is an active area of research these days. Many authors $[8,12,15,21,23,30]$ have worked in this area. The main aim of this paper is to establish an explicit formula for $S$-curvature of a homogeneous Finsler space with Randers change of square metric. The importance of S-curvature in RiemannFinsler geometry can be seen in several papers (e.g., [26, 27]).

Received July 19, 2019; accepted January 5, 2020.

2010 Mathematics Subject Classification. Primary 22E60; Secondary 53C30, 53C60.

${ }^{*}$ First author is very much thankful to UGC for providing financial assistance in terms of JRF fellowship via letter with Sr. No. 2061641032 and Ref. No. 19/06/2016(i)EU-V. Second author is thankful to Central University of Punjab, Bathinda for providing financial assistance as a Research Seed Money grant via the letter no. CUPB/CC/17/369. 
The simplest non-Riemannian metrics are the Randers metrics given by $F=$ $\alpha+\beta$ with $\|\beta\|_{\alpha}<1$, where $\alpha$ is a Riemannian metric and $\beta$ is a 1 -form. Besides Randers metrics, other interesting kind of non-Riemannian metrics are square metrics. Berwald's metric, constructed by Berwald [4] in 1929 as

$$
F=\frac{\left(\sqrt{\left(1-|x|^{2}\right)|y|^{2}+\langle x, y\rangle^{2}}+\langle x, y\rangle\right)^{2}}{\left(1-|x|^{2}\right)^{2} \sqrt{\left(1-|x|^{2}\right)|y|^{2}+\langle x, y\rangle^{2}}}
$$

is a classical example of square metric. Berwald's metric can be rewritten as follows:

$$
F=\frac{(\alpha+\beta)^{2}}{\alpha}
$$

where

$$
\alpha=\frac{\sqrt{\left(1-|x|^{2}\right)|y|^{2}+\langle x, y\rangle^{2}}}{\left(1-|x|^{2}\right)^{2}}
$$

and

$$
\beta=\frac{\langle x, y\rangle}{\left(1-|x|^{2}\right)^{2}} .
$$

An $(\alpha, \beta)$-metric expressed in the form (1.1) is called square metric [28]. Just as Randers metrics, square metrics play an important role in Finsler geometry. The importance of square metric can be seen in papers $[28,29,31])$. Square metrics can also be expressed in the form [31]

$$
F=\frac{\left(\sqrt{\left(1-b^{2}\right) \alpha^{2}+\beta^{2}}+\beta\right)^{2}}{\left(1-b^{2}\right)^{2} \sqrt{\left(1-b^{2}\right) \alpha^{2}+\beta^{2}}},
$$

where $b:=\left\|\beta_{x}\right\|_{\alpha}$ is the length of $\beta$.

In this case, $F=\alpha \phi\left(b^{2}, \frac{\beta}{\alpha}\right)$, where $\phi=\phi\left(b^{2}, s\right)$ is a smooth function, is called general $(\alpha, \beta)$-metric. If $\phi=\phi(s)$ is independent of $b^{2}$, then $F$ is called an $(\alpha, \beta)$-metric. An interesting fact is that if $\alpha=|y|$, and $\beta=\langle x, y\rangle$, then $F=|y| \phi\left(|x|^{2}, \frac{\langle x, y\rangle}{|y|}\right)$ becomes spherically symmetric metric.

If $F(\alpha, \beta)$ is a Finsler metric, then $F(\alpha, \beta) \longrightarrow \bar{F}(\alpha, \beta)$ is called a Randers change if

$$
\bar{F}(\alpha, \beta)=F(\alpha, \beta)+\beta .
$$

Above change of a Finsler metric has been introduced by M. Matsumoto [18], and it was named as "Randers change" by M. Hashiguchi and Y. Ichijyō [14]. In the current paper, we deal with Randers changed square metrics

$$
F=\frac{(\alpha+\beta)^{2}}{\alpha}+\beta=\alpha \phi(s), \text { where } \phi(s)=1+s^{2}+3 s .
$$


The paper is organized as follows:

In section 2, we discuss some basic definitions and results to be used in consequent sections. The existence of invariant vector fields on homogeneous Finsler spaces with Randers changed square metric has been proved in section 3 (see Theorem 3.1). Further, in section 4, we have established an explicit formula for $S$-curvature of afore said metric (see Theorem 4.2). Finally, in section 5, the mean Berwald curvature of this metrics has been calculated (see Theorem 5.1).

\section{Preliminaries}

First, we discuss some basic definitions and results required to study aforesaid spaces. We refer $[3,7,9]$ for notations and further details.

Definition 2.1. An n-dimensional real vector space $V$ is said to be a Minkowski space if there exists a real valued function $F: V \longrightarrow[0, \infty)$, called Minkowski norm, satisfying the following conditions:

- $F$ is smooth on $V \backslash\{0\}$,

- $F$ is positively homogeneous, i.e., $F(\lambda v)=\lambda F(v), \forall \lambda>0$,

- For any basis $\left\{u_{1}, u_{2}, \ldots, u_{n}\right\}$ of $V$ and $y=y^{i} u_{i} \in V$, the Hessian matrix $\left(g_{i j}\right)=\left(\frac{1}{2} F_{y^{i} y^{j}}^{2}\right)$ is positive-definite at every point of $V \backslash\{0\}$.

Definition 2.2. Let $M$ be a connected smooth manifold. If there exists a function $F: T M \longrightarrow[0, \infty)$ such that $F$ is smooth on the slit tangent bundle $T M \backslash\{0\}$ and the restriction of $F$ to any $T_{x} M, x \in M$, is a Minkowski norm, then $M$ is called a Finsler space and $F$ is called a Finsler metric.

An $(\alpha, \beta)$-metric on a connected smooth manifold $M$ is a Finsler metric $F$ constructed from a Riemannian metric $\alpha=\sqrt{a_{i j}(x) y^{i} y^{j}}$ and a one-form $\beta=b_{i}(x) y^{i}$ on $M$ and is of the form $F=\alpha \phi\left(\frac{\beta}{\alpha}\right)$, where $\phi$ is a smooth function on $M$. Basically, $(\alpha, \beta)$-metrics are the generalization of Randers metrics. Many authors $[12,15,21,22,24,30]$ have worked on $(\alpha, \beta)$-metrics. Let us recall Shen's lemma [7] which provides necessary and sufficient condition for an $(\alpha, \beta)$-metric to be a Finsler metric.

Lemma 2.1. Let $F=\alpha \phi(s), s=\beta / \alpha$, where $\phi$ is a smooth function on an open interval $\left(-b_{0}, b_{0}\right), \alpha$ is a Riemannian metric and $\beta$ is a 1-form with $\|\beta\|_{\alpha}<b_{0}$. Then $F$ is a Finsler metric if and only if the following conditions are satisfied:

$$
\phi(s)>0, \quad \phi(s)-s \phi^{\prime}(s)+\left(b^{2}-s^{2}\right) \phi^{\prime \prime}(s)>0, \quad \forall|s| \leq b<b_{0} .
$$

Before defining homogeneous Finsler spaces, we shall discuss some basic concepts below. 
Definition 2.3. Let $G$ be a smooth manifold having the structure of an abstract group. $G$ is called a Lie group, if the maps $i: G \longrightarrow G$ and $\mu: G \times G \longrightarrow G$ defined as $i(g)=g^{-1}$, and $\mu(g, h)=g h$ respectively, are smooth.

Let $G$ be a Lie group and $M$, a smooth manifold. Then a smooth map $f: G \times M \longrightarrow$ $M$ satisfying

$$
f\left(g_{2}, f\left(g_{1}, x\right)\right)=f\left(g_{2} g_{1}, x\right), \text { for all } g_{1}, g_{2} \in G, \quad \text { and } \quad x \in M
$$

is called a smooth action of $G$ on $M$.

Definition 2.4. Let $M$ be a smooth manifold and $G$, a Lie group. If $G$ acts smoothly on $M$, then $G$ is called a Lie transformation group of $M$.

The following theorem gives us a differentiable structure on the coset space of a Lie group.

Theorem 2.1. Let $G$ be a Lie group and $H$, its closed subgroup. Then there exists a unique differentiable structure on the left coset space $G / H$ with the induced topology that turns $G / H$ into a smooth manifold such that $G$ is a Lie transformation group of $G / H$.

Definition 2.5. Let $(M, F)$ be a connected Finsler space and $I(M, F)$ the group of isometries of $(M, F)$. If the action of $I(M, F)$ is transitive on $M$, then $(M, F)$ is said to be a homogeneous Finsler space.

Let $G$ be a Lie group acting transitively on a smooth manifold $M$. Then for $a \in M$, the isotropy subgroup $G_{a}$ of $G$ is a closed subgroup and by theorem 2.1, $G$ is a Lie transformation group of $G / G_{a}$. Further, $G / G_{a}$ is diffeomorphic to $M$.

Theorem 2.2. [9] Let $(M, F)$ be a Finsler space. Then $G=I(M, F)$, the group of isometries of $M$ is a Lie transformation group of $M$. Let $a \in M$ and $I_{a}(M, F)$ be the isotropy subgroup of $I(M, F)$ at a. Then $I_{a}(M, F)$ is compact.

Let $(M, F)$ be a homogeneous Finsler space, i.e., $G=I(M, F)$ acts transitively on $M$. For $a \in M$, let $H=I_{a}(M, F)$ be a closed isotropy subgroup of $G$ which is compact. Then $H$ is a Lie group itself being a closed subgroup of $G$. Write $M$ as the quotient space $G / H$.

Definition 2.6. [19] Let $\mathfrak{g}$ and $\mathfrak{h}$ be the Lie algebras of the Lie groups $G$ and $H$ respectively. Then the direct sum decomposition of $\mathfrak{g}$ as $\mathfrak{g}=\mathfrak{h}+\mathfrak{k}$, where $\mathfrak{k}$ is a subspace of $\mathfrak{g}$ such that $\operatorname{Ad}(h)(\mathfrak{k}) \subset \mathfrak{k} \forall h \in H$, is called a reductive decomposition of $\mathfrak{g}$, and if such decomposition exists, then $(G / H, F)$ is called reductive homogeneous space. 
Therefore, we can write, any homogeneous Finsler space as a coset space of a connected Lie group with an invariant Finsler metric. Here, the Finsler metric $F$ is viewed as $G$ invariant Finsler metric on $M$.

Definition 2.7. A one-parameter subgroup of a Lie group $G$ is a homomorphism $\psi: \mathbb{R} \longrightarrow G$, such that $\psi(0)=e$, where $e$ is the identity of $G$.

Recall [9] the following result which gives us the existence of one-parameter subgroup of a Lie group.

Theorem 2.3. Let $G$ be a Lie group having Lie algebra $\mathfrak{g}$. Then for any $Y \in \mathfrak{g}$, there exists a unique one-parameter subgroup $\psi$ such that $\dot{\psi}(0)=Y_{e}$, where e is the identity element of $G$.

Definition 2.8. Let $G$ be a Lie group with identity element $e$ and $\mathfrak{g}$ its Lie algebra. The exponential map exp $: \mathfrak{g} \longrightarrow G$ is defined by

$$
\exp (t Y)=\psi(t), \quad \forall t \in \mathbb{R},
$$

where $\psi: \mathbb{R} \longrightarrow G$ is unique one-parameter subgroup of $G$ with $\dot{\psi}(0)=Y_{e}$.

In case of reductive homogeneous manifold, we can identify the tangent space $T_{H}(G / H)$ of $G / H$ at the origin $e H=H$ with $\mathfrak{k}$ through the map

$$
\left.Y \longmapsto \frac{d}{d t} \exp (t X) H\right|_{t=0}, \quad Y \in \mathfrak{k},
$$

since $M$ is identified with $G / H$ and Lie algebra of any Lie group $G$ is viewed as $T_{e} G$.

\section{Invariant Vector Field}

For a homogeneous Finsler space with Randers changed square metric $F=$ $\frac{(\alpha+\beta)^{2}}{\alpha}+\beta$, in Theorem 3.1, we prove the existence of invariant vector field corresponding to 1-form $\beta$. For this, first we prove following lemmas:

Lemma 3.1. Let $(M, \alpha)$ be a Riemannian space and $\beta=b_{i} y^{i}$, a 1-form with $\|\beta\|=\sqrt{b_{i} b^{i}}<1$. Then the Randers changed square Finsler metric $F=\frac{(\alpha+\beta)^{2}}{\alpha}+\beta$, consists of a Riemannian metric $\alpha$ along with a smooth vector field $X$ on $M$ with $\alpha\left(\left.X\right|_{x}\right)<1, \forall x \in M$, i.e.,

$$
F(x, y)=\frac{\left(\alpha(x, y)+\left\langle\left. X\right|_{x}, y\right\rangle\right)^{2}}{\alpha(x, y)}+\left\langle\left. X\right|_{x}, y\right\rangle, \quad x \in M, \quad y \in T_{x} M,
$$

where $\langle$,$\rangle is the inner product induced by the Riemannian metric \alpha$. 
Proof. We know that the restriction of a Riemannian metric to a tangent space is an inner product. Therefore, the bilinear form $\langle u, v\rangle=a_{i j} u^{i} v^{j}, \quad u, v \in T_{x} M$ is an inner product on $T_{x} M$ for $x \in M$, and this inner product induces an inner product on $T_{x}^{*} M$, the cotangent space of $M$ at $x$ which gives us $\left\langle d x^{i}, d x^{j}\right\rangle=a^{i j}$. A linear isomorphism exists between $T_{x}^{*} M$ and $T_{x} M$, which can be defined by using this inner product. It follows that the 1 -form $\beta$ corresponds to a smooth vector field $X$ on $M$, which can be written as

$$
\left.X\right|_{x}=b^{i} \frac{\partial}{\partial x^{i}}, \text { where } b^{i}=a^{i j} b_{j} .
$$

Then, for $y \in T_{x} M$, we have

$$
\left\langle\left. X\right|_{x}, y\right\rangle=\left\langle b^{i} \frac{\partial}{\partial x^{i}}, y^{j} \frac{\partial}{\partial x^{j}}\right\rangle=b^{i} y^{j} a_{i j}=b_{j} y^{j}=\beta(y) .
$$

Also, we have

$$
\alpha^{2}(x, y)=a_{i j} y^{i} y^{j}
$$

which implies

$$
\alpha^{2}\left(\left.X\right|_{x}\right)=a_{i j} b^{i} b^{j}=\|\beta\|^{2}<1,
$$

i. e.,

$$
\alpha\left(\left.X\right|_{x}\right)<1 .
$$

This completes the proof.

Lemma 3.2. Let $(M, F)$ be a Finsler space with Randers changed square Finsler metric $F=\frac{(\alpha+\beta)^{2}}{\alpha}+\beta$. Let $I(M, F)$ be the group of isometries of $(M, F)$ and $I(M, \alpha)$ be that of Riemannian space $(M, \alpha)$. Then $I(M, F)$ is a closed subgroup of $I(M, \alpha)$.

Proof. Let $x \in M$ and $\phi:(M, F) \longrightarrow(M, F)$ be an isometry. Therefore, we have

$$
F(x, y)=F\left(\phi(x), d \phi_{x}(y)\right), \quad \forall y \in T_{x} M .
$$

By Lemma 3.1, we get

$$
\begin{aligned}
& \frac{\left(\alpha(x, y)+\left\langle\left. X\right|_{x}, y\right\rangle\right)^{2}}{\alpha(x, y)}+\left\langle\left. X\right|_{x}, y\right\rangle= \\
& =\frac{\left(\alpha\left(\phi(x), d \phi_{x}(y)\right)+\left\langle\left. X\right|_{\phi(x)}, d \phi_{x}(y)\right\rangle\right)^{2}}{\alpha\left(\phi(x), d \phi_{x}(y)\right)}+\left\langle\left. X\right|_{\phi(x)}, d \phi_{x}(y)\right\rangle,
\end{aligned}
$$

which gives us

$$
\begin{aligned}
& \alpha\left(\phi(x), d \phi_{x}(y)\right) \alpha^{2}(x, y)+\alpha\left(\phi(x), d \phi_{x}(y)\right)\left\langle\left. X\right|_{x}, y\right\rangle^{2} \\
& +3 \alpha\left(\phi(x), d \phi_{x}(y)\right) \alpha(x, y)\left\langle\left. X\right|_{x}, y\right\rangle \\
& =\alpha(x, y) \alpha^{2}\left(\phi(x), d \phi_{x}(y)\right)+\alpha(x, y)\left\langle\left. X\right|_{\phi(x)}, d \phi_{x}(y)\right\rangle^{2} \\
& +3 \alpha(x, y) \alpha\left(\phi(x), d \phi_{x}(y)\right)\left\langle\left. X\right|_{\phi(x)}, d \phi_{x}(y)\right\rangle
\end{aligned}
$$


Replacing $y$ by $-y$ in equation (3.1), we get

$$
\begin{aligned}
& \alpha\left(\phi(x), d \phi_{x}(y)\right) \alpha^{2}(x, y)+\alpha\left(\phi(x), d \phi_{x}(y)\right)\left\langle\left. X\right|_{x}, y\right\rangle^{2} \\
& -3 \alpha\left(\phi(x), d \phi_{x}(y)\right) \alpha(x, y)\left\langle\left. X\right|_{x}, y\right\rangle \\
& =\alpha(x, y) \alpha^{2}\left(\phi(x), d \phi_{x}(y)\right)+\alpha(x, y)\left\langle\left. X\right|_{\phi(x)}, d \phi_{x}(y)\right\rangle^{2} \\
& -3 \alpha(x, y) \alpha\left(\phi(x), d \phi_{x}(y)\right)\left\langle\left. X\right|_{\phi(x)}, d \phi_{x}(y)\right\rangle
\end{aligned}
$$

Subtracting equation (3.2) from equation (3.1), we get

$$
\alpha\left(\phi(x), d \phi_{x}(y)\right) \alpha(x, y)\left\langle\left. X\right|_{x}, y\right\rangle=\alpha(x, y) \alpha\left(\phi(x), d \phi_{x}(y)\right)\left\langle\left. X\right|_{\phi(x)}, d \phi_{x}(y)\right\rangle,
$$

which implies

$$
\left\langle\left. X\right|_{x}, y\right\rangle=\left\langle\left. X\right|_{\phi(x)}, d \phi_{x}(y)\right\rangle .
$$

Adding equations (3.1) and (3.2) and using equation (3.3), we get

$$
\begin{aligned}
& \alpha\left(\phi(x), d \phi_{x}(y)\right) \alpha^{2}(x, y)+\alpha\left(\phi(x), d \phi_{x}(y)\right)\left\langle\left. X\right|_{x}, y\right\rangle^{2} \\
& =\alpha(x, y) \alpha^{2}\left(\phi(x), d \phi_{x}(y)\right)+\alpha(x, y)\left\langle\left. X\right|_{x}, y\right\rangle^{2},
\end{aligned}
$$

which leads to

$$
\alpha(x, y)=\alpha\left(\phi(x), d \phi_{x}(y)\right) .
$$

Therefore $\phi$ is an isometry with respect to the Riemannian metric $\alpha$ and $d \phi_{x}\left(\left.X\right|_{x}\right)=$ $\left.X\right|_{\phi(x)}$. Thus $I(M, F)$ is a closed subgroup of $I(M, \alpha)$.

From Lemma (3.2), we conclude that if $(M, F)$ is a homogeneous Finsler space with Randers change of square metric $F=\frac{(\alpha+\beta)^{2}}{\alpha}+\beta$, then the Riemannian space $(M, \alpha)$ is homogeneous. Further, $M$ can be written as a coset space $G / H$, where $G=I(M, F)$ is a Lie transformation group of $M$ and $H$, the compact isotropy subgroup $I_{a}(M, F)$ of $I(M, F)$ at some point $a \in M[10]$. Let $\mathfrak{g}$ and $\mathfrak{h}$ be the Lie algebras of the Lie groups $G$ and $H$ respectively. If $\mathfrak{g}$ can be written as a direct sum of subspaces $\mathfrak{h}$ and $\mathfrak{k}$ of $\mathfrak{g}$ such that $\operatorname{Ad}(h) \mathfrak{k} \subset \mathfrak{k} \forall h \in H$, then from definition 2.6, $(G / H, F)$ is a reductive homogeneous space.

Therefore, we can write, any homogeneous Finsler space as a coset space of a connected Lie group with an invariant Finsler metric. Here, the Finsler metric $F$ is viewed as $G$ invariant Finsler metric on $M$.

Theorem 3.1. Let $F=\frac{(\alpha+\beta)^{2}}{\alpha}+\beta$ be a G-invariant Randers changed square metric on $G / H$. Then $\alpha$ is a $G$-invariant Riemannian metric and the vector field $X$ corresponding to the 1 -form $\beta$ is also $G$-invariant. 
Proof. Since $F$ is a $G$-invariant metric on $G / H$, we have

$$
F(y)=F(\operatorname{Ad}(h) y), \quad \forall h \in H, \quad y \in \mathfrak{k} .
$$

By Lemma 3.1, we get

$$
\frac{(\alpha(y)+\langle X, y\rangle)^{2}}{\alpha(y)}+\langle X, y\rangle=\frac{(\alpha(\operatorname{Ad}(h) y)+\langle X, \operatorname{Ad}(h) y\rangle)^{2}}{\alpha(\operatorname{Ad}(h) y)}+\langle X, \operatorname{Ad}(h) y\rangle .
$$

After simplification, we get

$$
\begin{gathered}
\alpha(\operatorname{Ad}(h) y) \alpha^{2}(y)+\alpha(\operatorname{Ad}(h) y)\langle X, y\rangle^{2}+3 \alpha(\operatorname{Ad}(h) y) \alpha(y)\langle X, y\rangle \\
(3.5)=\alpha(y) \alpha^{2}(\operatorname{Ad}(h) y)+\alpha(y)\langle X, \operatorname{Ad}(h) y\rangle^{2}+3 \alpha(y) \alpha(\operatorname{Ad}(h) y)\langle X, \operatorname{Ad}(h) y\rangle .
\end{gathered}
$$

Replacing $y$ by $-y$ in equation (3.5), we get

$$
\begin{gathered}
\alpha(\operatorname{Ad}(h) y) \alpha^{2}(y)+\alpha(\operatorname{Ad}(h) y)\langle X, y\rangle^{2}-3 \alpha(\operatorname{Ad}(h) y) \alpha(y)\langle X, y\rangle \\
(3.6)=\alpha(y) \alpha^{2}(\operatorname{Ad}(h) y)+\alpha(y)\langle X, \operatorname{Ad}(h) y\rangle^{2}-3 \alpha(y) \alpha(\operatorname{Ad}(h) y)\langle X, \operatorname{Ad}(h) y\rangle .
\end{gathered}
$$

Subtracting equation (3.6) from equation (3.5), we get

$$
\alpha(\operatorname{Ad}(h) y) \alpha(y)\langle X, y\rangle=\alpha(y) \alpha(\operatorname{Ad}(h) y)\langle X, \operatorname{Ad}(h) y\rangle,
$$

which gives us

$$
\langle X, y\rangle=\langle X, \operatorname{Ad}(h) y\rangle \text {. }
$$

Adding equations (3.5) and (3.6) and using equation (3.7), we get

$$
\alpha(\operatorname{Ad}(h) y) \alpha^{2}(y)+\alpha(\operatorname{Ad}(h) y)\langle X, y\rangle^{2}=\alpha(y) \alpha^{2}(\operatorname{Ad}(h) y)+\alpha(y)\langle X, y\rangle^{2}
$$

which leads to

$$
\alpha(y)=\alpha(\operatorname{Ad}(h) y)
$$

Therefore, $\alpha$ is a $G$-invariant Riemannian metric and $\operatorname{Ad}(h) X=X$, which proves that $X$ is also $G$-invariant.

The following theorem gives us a complete description of invariant vector fields.

Theorem 3.2. [11] There exists a bijection between the set of invariant vector fields on $G / H$ and the subspace

$$
V=\{Y \in \mathfrak{k}: A d(h) Y=Y, \forall h \in H\} .
$$




\section{S-curvature of homogeneous Finsler space with}

Now, we discuss $S$-curvature, a quantity used to measure the rate of change of the volume form of a Finsler space along geodesics. Let $V$ be an n-dimensional real vector space having a basis $\left\{\alpha_{i}\right\}$ and $F$ be a Minkowski norm on $V$. Let $V$ ol $B$ to be the volume of a subset $B$ of $\mathbb{R}^{n}$, and $B^{n}$ be the open unit ball. The function $\tau=\tau(y)$ defined as

$$
\tau(y)=\ln \left(\frac{\sqrt{\operatorname{det}\left(g_{i j}(y)\right)}}{\sigma_{F}}\right), \quad y \in V-\{0\}
$$

where

$$
\sigma_{F}=\frac{\operatorname{Vol}\left(B^{n}\right)}{\operatorname{Vol}\left\{\left(y^{i}\right) \in \mathbb{R}^{n}: F\left(y^{i} \alpha_{i}\right)<1\right\}},
$$

is called the distortion of $(V, F)$.

For a Finsler space $(M, F), \tau=\tau(x, y)$ is the distortion of Minkowski norm $F_{x}$ on $T_{x} M, x \in M$. Let $\gamma$ be a geodesic with $\gamma(0)=x, \dot{\gamma}(0)=y$, where $y \in T_{x} M$, then $S$-curvature denoted as $S(x, y)$ is the rate of change of distortion along the geodesic $\gamma$, i.e.,

$$
S(x, y)=\left.\frac{d}{d t}\{\tau(\gamma(t), \dot{\gamma}(t))\}\right|_{t=0} .
$$

Here, it is to be noted that $S(x, y)$ is positively homogeneous of degree one, i.e., for $\lambda>0$, we have $S(x, \lambda y)=\lambda S(x, y)$.

$S$-curvature of a Finsler space is related to a volume form. There are two important volume forms in Finsler geometry: the Busemann-Hausdorff volume form $d V_{B H}=\sigma_{B H}(x) d x$ and the Holmes-Thompson volume form $d V_{H T}=\sigma_{H T}(x) d x$ defined respectively as

$$
\sigma_{B H}(x)=\frac{\operatorname{Vol}\left(B^{n}\right)}{\operatorname{Vol} A}
$$

and

$$
\sigma_{H T}(x)=\frac{1}{\operatorname{Vol}\left(B^{n}\right)} \int_{A} \operatorname{det}\left(g_{i j}\right) d y
$$

where $A=\left\{\left(y^{i}\right) \in \mathbb{R}^{n}: F\left(x, y^{i} \frac{\partial}{\partial x^{i}}\right)<1\right\}$.

If the Finsler metric $F$ is replaced by a Riemannian metric, then both the volume forms reduce to a single Riemannian volume form $d V_{H T}=d V_{B H}=\sqrt{\operatorname{det}\left(g_{i j}(x)\right)} d x$.

Next, for the function

$$
T(s)=\phi\left(\phi-s \phi^{\prime}\right)^{n-2}\left\{\left(\phi-s \phi^{\prime}\right)+\left(b^{2}-s^{2}\right) \phi^{\prime \prime}\right\}
$$


the volume form $d V=d V_{B H}$ or $d V_{H T}$ is given by $d V=f(b) d V_{\alpha}$, where

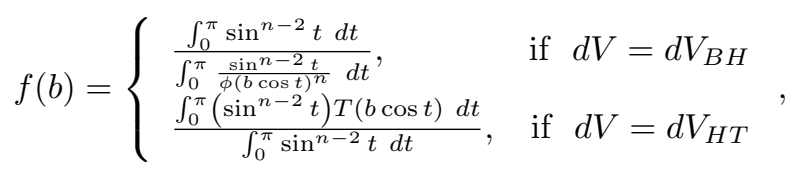

and $d V_{\alpha}=\sqrt{\operatorname{det}\left(a_{i j}\right)} d x$ is the Riemannian volume form of $\alpha$.

The formula for $S$-curvature of an $(\alpha, \beta)$-metric, in local co-ordinate system, introduced by Cheng and Shen [5], is as follows:

$$
S=\left(2 \psi-\frac{f^{\prime}(b)}{b f(b)}\right)\left(r_{0}+s_{o}\right)-\frac{\Phi}{2 \alpha \Delta^{2}}\left(r_{00}-2 \alpha Q s_{0}\right),
$$

where

$$
\begin{aligned}
Q & =\frac{\phi^{\prime}}{\phi-s \phi^{\prime}}, \\
\Delta & =1+s Q+\left(b^{2}-s^{2}\right) Q^{\prime}, \\
\psi & =\frac{Q^{\prime}}{2 \Delta}, \\
\Phi & =\left(s Q^{\prime}-Q\right)(n \Delta+1+s Q)-\left(b^{2}-s^{2}\right)(1+s Q) Q^{\prime \prime}, \\
r_{i j} & =\frac{1}{2}\left(b_{i \mid j}+b_{j \mid i}\right), r_{j}=b^{i} r_{i j}, r_{0}=r_{i} y^{i}, r_{00}=r_{i j} y^{i} y^{j}, \\
s_{i j} & =\frac{1}{2}\left(b_{i \mid j}-b_{j \mid i}\right), s_{j}=b^{i} s_{i j}, s_{0}=s_{i} y^{i} .
\end{aligned}
$$

It is well known [5] that if the Riemannian length $b$ is constant, then $r_{0}+s_{0}=0$. Therefore, in this case, the equation (4.1) takes the form

$$
S=-\frac{\Phi}{2 \alpha \Delta^{2}}\left(r_{00}-2 \alpha Q s_{0}\right) \text {. }
$$

After Shen's work on $S$-curvature, Cheng and Shen [5] characterized Finsler metrics with isotropic $S$-curvature in 2009. In the same year, Deng [8] gave an explicit formula for $S$-curvature of homogeneous Randers spaces and he proved that a homogeneous Randers space having almost isotropic $S$-curvature has vanishing $S$-curvature. Later in 2010, Deng and Wang [12] gave a formula for $S$-curvature of homogeneous $(\alpha, \beta)$-metrics. They also derived a formula for mean Berwald curvature $E_{i j}$ of Randers metric. Recently, Shanker and Kaur [22] have proved that there is a mistake in the formula of $S$-curvature given in [12], and they have given the correct version of the formula for $S$-curvature of homogeneous $(\alpha, \beta)$-metrics. Further, some progress has been done in the study of $S$-curvature of homogeneous Finsler spaces (see $[15,30]$ for detail). 
Definition 4.1. Let $(M, F)$ be an $n$-dimensional Finsler space. If there exists a smooth function $c(x)$ on $M$ and a closed 1-form $\omega$ such that

$$
S(x, y)=(n+1)(c(x) F(y)+\omega(y)), \quad x \in M, y \in T_{x}(M),
$$

then $(M, F)$ is said to have almost isotropic $S$-curvature. In addition, if $\omega$ is zero, then $(M, F)$ is said to have isotropic $S$-curvature.

Also, if $\omega$ is zero and $c(x)$ is constant, then we say, $(M, F)$ has constant $S$-curvature.

With above notations, let us recall the following theorem:

Theorem 4.1. [22] Let $F=\alpha \phi(s)$ be a $G$-invariant $(\alpha, \beta)$-metric on the reductive homogeneous Finsler space $G / H$ with a decomposition of the Lie algebra $\mathfrak{g}=\mathfrak{h}+\mathfrak{k}$. Then the $S$-curvature is given by

$$
S(H, y)=\frac{\Phi}{2 \alpha \Delta^{2}}\left(\left\langle[v, y]_{\mathfrak{k}}, y\right\rangle+\alpha Q\left\langle[v, y]_{\mathfrak{k}}, v\right\rangle\right),
$$

where $v \in \mathfrak{k}$ corresponds to the 1 -form $\beta$ and $\mathfrak{k}$ is identified with the tangent space $T_{H}(G / H)$ of $G / H$ at the origin $H$.

Now, we establish a formula for S-curvature of a homogeneous Finsler space with Randers changed square metric.

Theorem 4.2. Let $G / H$ be reductive homogeneous Finsler space with a decomposition of the Lie algebra $\mathfrak{g}=\mathfrak{h}+\mathfrak{k}$, and $F=\frac{(\alpha+\beta)^{2}}{\alpha}+\beta$ be a $G$-invariant Randers changed square metric on $G / H$. Then the $S$-curvature is given by

$$
\begin{aligned}
S(H, y)= & {\left[\begin{array}{c}
-12 s^{5} n+(-27 n+9) s^{4}+\left(8 n b^{2}+4 n-4 b^{2}+16\right) s^{3} \\
+\left(18 n b^{2}+18 n-18 b^{2}+18\right) s^{2}+-12 b^{2} s-3-6 b^{2}-6 n b^{2}-3 n \\
2\left(-3 s^{2}+1+2 b^{2}\right)\left(1-2 s^{2}-3 s^{4}+3 s-9 s^{3}+2 b^{2}+2 b^{2} s^{2}+6 b^{2} s\right)
\end{array}\right] \times } \\
& \left(\frac{2 s+3}{1-s^{2}}\left\langle[v, y]_{\mathfrak{k}}, v\right\rangle+\frac{1}{\alpha}\left\langle[v, y]_{\mathfrak{k}}, y\right\rangle\right)
\end{aligned}
$$

where $v \in \mathfrak{k}$ corresponds to the 1 -form $\beta$ and $\mathfrak{k}$ is identified with the tangent space $T_{H}(G / H)$ of $G / H$ at the origin $H$.

Proof. For Randers changed square metric

$$
F=\alpha \phi(s), \text { where } \phi(s)=1+s^{2}+3 s
$$


the entities written in the equation (4.1) take the values as follows:

$$
\begin{aligned}
Q & =\frac{\phi^{\prime}}{\phi-s \phi^{\prime}}=\frac{2 s+3}{1-s^{2}} \\
Q^{\prime} & =\frac{2 s^{2}+6 s+2}{\left(1-s^{2}\right)^{2}} \\
Q^{\prime \prime} & =\frac{4 s^{3}+18 s^{2}+12 s+6}{\left(1-s^{2}\right)^{3}}, \\
\Delta & =1+s Q+\left(b^{2}-s^{2}\right) Q^{\prime} \\
& =1+s\left(\frac{2 s+3}{1-s^{2}}\right)+\left(b^{2}-s^{2}\right) \frac{2 s^{2}+6 s+2}{\left(1-s^{2}\right)^{2}} \\
& =\frac{-3 s^{4}-9 s^{3}+\left(2 b^{2}-2\right) s^{2}+\left(6 b^{2}+3\right) s+2 b^{2}+1}{\left(1-s^{2}\right)^{2}},
\end{aligned}
$$

$\Phi=\left(s Q^{\prime}-Q\right)(1+n \Delta+s Q)+\left(s^{2}-b^{2}\right)(1+s Q) Q^{\prime \prime}$

$$
\begin{aligned}
= & \left(\frac{2 s^{3}+6 s^{2}+2 s}{\left(1-s^{2}\right)^{2}}-\frac{2 s+3}{1-s^{2}}\right) \times \\
& \left\{1+\frac{-3 n s^{4}-9 n s^{3}+\left(2 n b^{2}-2 n\right) s^{2}+\left(6 n b^{2}+3 n\right) s+2 n b^{2}+n}{\left(1-s^{2}\right)^{2}}+\frac{2 s^{2}+3 s}{1-s^{2}}\right\}
\end{aligned}
$$$$
+\left(s^{2}-b^{2}\right)\left\{1+\frac{2 s^{2}+3 s}{1-s^{2}}\right\}\left(\frac{4 s^{3}+18 s^{2}+12 s+6}{\left(1-s^{2}\right)^{3}}\right)
$$

$$
\begin{aligned}
= & \frac{1}{\left(1-s^{2}\right)^{4}}\left\{-(12 n+4) s^{7}-(63 n+21) s^{6}+\left(8 n b^{2}-89 n-27\right) s^{5}\right. \\
& +\left(42 n b^{2}+3 n+15\right) s^{4}+\left(62 n b^{2}+58 n+40\right) s^{3}+\left(12 n b^{2}+15 n+9\right) s^{2}
\end{aligned}
$$$$
\left.-\left(18 n b^{2}+9 n+9\right) s-\left(6 n b^{2}+3 n+3\right)\right\}+\frac{1}{\left(1-s^{2}\right)^{4}}\left\{4 s^{7}+30 s^{6}+\left(70-4 b^{2}\right) s^{5}\right.
$$$$
\left.+\left(60-30 b^{2}\right) s^{4}+\left(30-70 b^{2}\right) s^{3}+\left(6-60 b^{2}\right) s^{2}-30 b^{2} s-6 b^{2}\right\} .
$$$$
=\frac{1}{\left(1-s^{2}\right)^{4}}\left\{-12 n s^{7}+(9-63 n) s^{6}+\left(8 n b^{2}-4 b^{2}-89 n+43\right) s^{5}\right.
$$$$
+\left(42 n b^{2}-30 b^{2}+3 n+75\right) s^{4}+\left(62 n b^{2}-70 b^{2}+58 n+70\right) s^{3}
$$$$
+\left(12 n b^{2}-60 b^{2}+15 n+15\right) s^{2}-\left(18 n b^{2}+30 b^{2}+9 n+9\right) s
$$$$
\left.-\left(6 n b^{2}+6 b^{2}+3 n+3\right)\right\}
$$

After substituting these values in equation (4.3), we get the formula 4.4 for $S$ curvature of homogeneous Finsler space with Randers changed square metric.

Corollary 4.1. Let $G / H$ be reductive homogeneous Finsler space with a decomposition of the Lie algebra $\mathfrak{g}=\mathfrak{h}+\mathfrak{k}$, and $F=\frac{(\alpha+\beta)^{2}}{\alpha}+\beta$ be a G-invariant Randers 
changed square metric on $G / H$. Then $(G / H, F)$ has isotropic $S$-curvature if and only if it has vanishing $S$-curvature.

Proof. Converse part is obvious. For necessary part, suppose $G / H$ has isotropic $S$-curvature, then

$$
S(x, y)=(n+1) c(x) F(y), \quad x \in G / H, y \in T_{x}(G / H) .
$$

Taking $x=H$ and $y=v$ in the equation (4.4), we get $c(H)=0$.

Consequently $S(H, y)=0 \forall y \in T_{H}(G / H)$.

Since $F$ is a homogeneous metric, we have $S=0$ everywhere.

\section{Mean Berwald Curvature}

There is another quantity [7] associated with $S$-curvature called Mean Berwald curvature.

Let $E_{i j}=\frac{1}{2} \frac{\partial^{2}}{\partial y^{2} \partial y^{j}} S(x, y)=\frac{1}{2} \frac{\partial^{2}}{\partial y^{2} \partial y^{j}}\left(\frac{\partial G^{m}}{\partial y^{m}}\right)(x, y)$, where $G^{m}$ are spray coefficients. Then $\mathcal{E}:=E_{i j} d x^{i} \otimes d x^{j}$ is a tensor on $T M \backslash\{0\}$, which we call $E$ tensor. $E$ tensor can also be viewed as a family of symmetric forms $E_{y}: T_{x} M \times T_{x} M \longrightarrow \mathbb{R}$ defined as

$$
E_{y}(u, v)=E_{i j}(x, y) u^{i} v^{j}
$$

where $u=\left.u^{i} \frac{\partial}{\partial x^{i}}\right|_{x}, \quad v=\left.v^{i} \frac{\partial}{\partial x^{i}}\right|_{x} \in T_{x} M$. Then the collection $\left\{E_{y}: y \in T M \backslash\{0\}\right\}$ is called $E$-curvature or mean Berwald curvature.

In this section, we calculate the mean Berwald curvature of a homogeneous Finsler space with the aforesaid metrics. To calculate it, we need the following:

At the origin, $a_{i j}=\delta_{j}^{i}$,

therefore $y_{i}=a_{i j} y^{j}=\delta_{j}^{i} y^{j}=y^{i}$,

$$
\begin{aligned}
\alpha_{y^{i}} & =\frac{y_{i}}{\alpha} \\
\beta_{y^{i}} & =b_{i} \\
s_{y^{i}} & =\frac{\partial}{\partial y^{i}}\left(\frac{\beta}{\alpha}\right)=\frac{b_{i} \alpha-s y_{i}}{\alpha^{2}}, \\
s_{y^{i} y^{j}} & =\frac{\partial}{\partial y^{j}}\left(\frac{b_{i} \alpha-s y_{i}}{\alpha^{2}}\right) \\
& =\frac{\alpha^{2}\left\{b_{i} \frac{y_{j}}{\alpha}-\left(\frac{b_{j} \alpha-s y_{j}}{\alpha^{2}}\right) y_{i}-s \delta_{j}^{i}\right\}-\left(b_{i} \alpha-s y_{i}\right) 2 \alpha \frac{y_{j}}{\alpha}}{\alpha^{4}} \\
& =\frac{-\left(b_{i} y_{j}+b_{j} y_{i}\right) \alpha+3 s y_{i} y_{j}-\alpha^{2} s \delta_{j}^{i}}{\alpha^{4}},
\end{aligned}
$$

Assuming

$$
\begin{aligned}
& -12 s^{5} n+(-27 n+9) s^{4}+\left(8 n b^{2}+4 n-4 b^{2}+16\right) s^{3} \\
& \quad \frac{+\left(18 n b^{2}+18 n-18 b^{2}+18\right) s^{2}+-12 b^{2} s-3-6 b^{2}-6 n b^{2}-3 n}{2\left(-3 s^{2}+1+2 b^{2}\right)\left(1-2 s^{2}-3 s^{4}+3 s-9 s^{3}+2 b^{2}+2 b^{2} s^{2}+6 b^{2} s\right)}=B
\end{aligned}
$$


in the equation (4.4), we find

$$
\begin{aligned}
\frac{\partial B}{\partial y^{j}}= & \frac{1}{2}\left(-3 s^{2}+1+2 b^{2}\right)^{-1}\left(-3 s^{4}-9 s^{3}+\left(2 b^{2}-2\right) s^{2}+\left(3+6 b^{2}\right) s+1+2 b^{2}\right)^{-2} \times \\
& \left\{-36 n s^{8}+(-162 n+54) s^{7}+\left(-207 n-36 b^{2}+225\right) s^{6}\right. \\
& +\left(-252 b^{2}+90 n-36 n b^{2}+522\right) s^{5} \\
& +\left(-488 b^{2}+631-80 n b^{2}+199 n-8 b^{4}+16 n b^{4}\right) s^{4} \\
& +\left(-30 n+186+96 n b^{4}-408 b^{2}-120 n b^{2}-48 b^{4}\right) s^{3} \\
& +\left(-228 b^{2}+156 n b^{4}-108 b^{4}-33-60 n b^{2}-69 n\right) s^{2} \\
& +\left(96 n b^{4}+6 n+6-48 b^{4}-12 b^{2}+60 n b^{2}\right) s \\
& \left.+9+24 b^{2}+36 n b^{4}+12 b^{4}+36 n b^{2}+9 n\right\} s_{y^{j}},
\end{aligned}
$$

and

$$
\begin{aligned}
\frac{\partial^{2} B}{\partial y^{i} \partial y^{j}}= & \frac{1}{2} \frac{\partial}{\partial y^{i}}\left[\left(-3 s^{2}+1+2 b^{2}\right)^{-1}\left(-3 s^{4}-9 s^{3}+\left(2 b^{2}-2\right) s^{2}+\left(3+6 b^{2}\right) s+1+2 b^{2}\right)^{-2} \times\right. \\
& \left\{-36 n s^{8}(-162 n+54) s^{7}+\left(-207 n-36 b^{2}+225\right) s^{6}\right. \\
& +\left(-252 b^{2}+90 n-36 n b^{2}+522\right) s^{5} \\
& +\left(-488 b^{2}+631-80 n b^{2}+199 n-8 b^{4}+16 n b^{4}\right) s^{4} \\
& +\left(-30 n+186+96 n b^{4}-408 b^{2}-120 n b^{2}-48 b^{4}\right) s^{3} \\
& +\left(-228 b^{2}+156 n b^{4}-108 b^{4}-33-60 n b^{2}-69 n\right) s^{2} \\
& +\left(96 n b^{4}+6 n+6-48 b^{4}-12 b^{2}+60 n b^{2}\right) s \\
& \left.\left.+9+24 b^{2}+36 n b^{4}+12 b^{4}+36 n b^{2}+9 n\right\}\right] s_{y^{j}} \\
+ & \frac{1}{2}\left(-3 s^{2}+1+2 b^{2}\right)^{-1}\left(-3 s^{4}-9 s^{3}+\left(2 b^{2}-2\right) s^{2}+\left(3+6 b^{2}\right) s+1+2 b^{2}\right)^{-2} \times \\
& \left\{-36 n s^{8}+(-162 n+54) s^{7}+\left(-207 n-36 b^{2}+225\right) s^{6}\right. \\
& +\left(-252 b^{2}+90 n-36 n b^{2}+522\right) s^{5} \\
+ & \left(-488 b^{2}+631-80 n b^{2}+199 n-8 b^{4}+16 n b^{4}\right) s^{4} \\
+ & \left(-30 n+186+96 n b^{4}-408 b^{2}-120 n b^{2}-48 b^{4}\right) s^{3} \\
+ & \left(-228 b^{2}+156 n b^{4}-108 b^{4}-33-60 n b^{2}-69 n\right) s^{2} \\
+ & \left(96 n b^{4}+6 n+6-48 b^{4}-12 b^{2}+60 n b^{2}\right) s \\
+ & \left.9+24 b^{2}+36 n b^{4}+12 b^{4}+36 n b^{2}+9 n\right\} s_{y^{i} y^{j}} \\
& \\
& \\
&
\end{aligned}
$$




$$
\begin{aligned}
= & \left(-3 s^{2}+1+2 b^{2}\right)^{-1}\left(-3 s^{4}-9 s^{3}+\left(2 b^{2}-2\right) s^{2}+\left(3+6 b^{2}\right) s+1+2 b^{2}\right)^{-3} \times \\
& \left\{-108 s^{11} n+(243-729 n) s^{10}+\left(1593-216 b^{2}-144 n b^{2}-1935 n\right) s^{9}\right. \\
& +\left(5940-2052 b^{2}-1404 n b^{2}-1512 n\right) s^{8} \\
& +\left(-144 b^{4}-6570 b^{2}+144 n b^{4}+1440 n-4338 n b^{2}+13356\right) s^{7} \\
& +\left(15894-10188 b^{2}+1260 n b^{4}+1638 n-1332 b^{4}-6300 n b^{2}\right) s^{6} \\
& +\left(8706-4884 b^{4}-4254 n b^{2}-1122 n-8574 b^{2}+3756 n b^{4}\right) s^{5} \\
& +\left(3132-7560 b^{4}-1080 n+5400 n b^{4}-3834 b^{2}+54 n b^{2}\right) s^{4} \\
& +\left(2634 n b^{2}+3960 n b^{4}+1700-4680 b^{4}+40 b^{6}+40 n b^{6}+332 n+402 b^{2}\right) s^{3} \\
& +\left(1476 n b^{4}+1368 n b^{2}+720 b^{2}+315 n-1116 b^{4}+639\right) s^{2} \\
& +\left(-90 n b^{2}-15 n-162 b^{2}-180 n b^{4}+21-468 b^{4}-120 n b^{6}-120 b^{6}\right) s \\
& \left.-24-24 n-120 n b^{6}-120 b^{6}-126 b^{2}-216 n b^{4}-126 n b^{2}-216 b^{4}\right\} s_{y^{i}} s_{y^{j}} \\
+ & \frac{1}{2}\left(-3 s^{2}+1+2 b^{2}\right)^{-1}\left(-3 s^{4}-9 s^{3}+\left(2 b^{2}-2\right) s^{2}+\left(3+6 b^{2}\right) s+1+2 b^{2}\right)^{-2} \times \\
& \left\{-36 n s^{8}+(-162 n+54) s^{7}+\left(-207 n-36 b^{2}+225\right) s^{6}\right. \\
& +\left(-252 b^{2}+90 n-36 n b^{2}+522\right) s^{5} \\
& +\left(-488 b^{2}+631-80 n b^{2}+199 n-8 b^{4}+16 n b^{4}\right) s^{4} \\
& +\left(-30 n+186+96 n b^{4}-408 b^{2}-120 n b^{2}-48 b^{4}\right) s^{3} \\
& +\left(-228 b^{2}+156 n b^{4}-108 b^{4}-33-60 n b^{2}-69 n\right) s^{2} \\
& +\left(96 n b^{4}+6 n+6-48 b^{4}-12 b^{2}+60 n b^{2}\right) s \\
+ & \left.+24 b^{2}+36 n b^{4}+12 b^{4}+36 n b^{2}+9 n\right\} s_{y^{i} y^{j}}
\end{aligned}
$$

Theorem 5.1. Let $G / H$ be a reductive homogeneous Finsler space with a decomposition of the Lie algebra $\mathfrak{g}=\mathfrak{h}+\mathfrak{k}$, and $F=\frac{(\alpha+\beta)^{2}}{\alpha}+\beta$ be a $G$-invariant Randers changed square metric on $G / H$. Then the mean Berwald curvature of the homogeneous Finsler space with Randers changed square metric is given by

$$
\begin{aligned}
E_{i j}(H, y)= & \frac{1}{2}\left[\left(\frac{1}{\alpha} \frac{\partial^{2} B}{\partial y^{i} \partial y^{j}}-\frac{y_{i}}{\alpha^{3}} \frac{\partial B}{\partial y^{j}}-\frac{y_{j}}{\alpha^{3}} \frac{\partial B}{\partial y^{i}}-\frac{B}{\alpha^{3}} \delta_{i}^{j}+\frac{3 B}{\alpha^{5}} y_{i} y_{j}\right)\left\langle[v, y]_{\mathfrak{k}}, y\right\rangle\right. \\
& +\left(\frac{1}{\alpha} \frac{\partial B}{\partial y^{j}}-\frac{B}{\alpha^{3}} y_{j}\right)\left(\left\langle\left[v, v_{i}\right]_{\mathfrak{k}}, y\right\rangle+\left\langle[v, y]_{\mathfrak{k}}, v_{i}\right\rangle\right) \\
& +\left(\frac{1}{\alpha} \frac{\partial B}{\partial y^{i}}-\frac{B}{\alpha^{3}} y_{i}\right)\left(\left\langle\left[v, v_{j}\right]_{\mathfrak{k}}, y\right\rangle+\left\langle[v, y]_{\mathfrak{k}}, v_{j}\right\rangle\right)
\end{aligned}
$$


688

$$
\begin{aligned}
& +\frac{B}{\alpha}\left(\left\langle\left[v, v_{j}\right]_{\mathfrak{k}}, v_{i}\right\rangle+\left\langle\left[v, v_{i}\right]_{\mathfrak{k}}, v_{j}\right\rangle\right) \\
& +\left\{\frac{2 s+3}{1-s^{2}} \frac{\partial^{2} B}{\partial y^{i} \partial y^{j}}+\frac{2 s^{2}+6 s+2}{\left(1-s^{2}\right)^{2}} s_{y^{i}} \frac{\partial B}{\partial y^{j}}+\frac{2 s^{2}+6 s+2}{\left(1-s^{2}\right)^{2}} s_{y^{j}} \frac{\partial B}{\partial y^{i}}\right. \\
& \left.+\frac{\left(4 s^{3}+18 s^{2}+12 s+6\right) B}{\left(1-s^{2}\right)^{3}} s_{y^{i}} s_{y^{j}}+\frac{\left(2 s^{2}+6 s+2\right) B}{\left(1-s^{2}\right)^{2}} s_{y^{i} y^{j}}\right\}\left\langle[v, y]_{\mathfrak{k}}, v\right\rangle \\
& +\left\{\frac{2 s+3}{1-s^{2}} \frac{\partial B}{\partial y^{j}}+\frac{\left(2 s^{2}+6 s+2\right) B}{\left(1-s^{2}\right)^{2}} s_{y^{j}}\right\}\left\langle\left[v, v_{i}\right]_{\mathfrak{k}}, v\right\rangle \\
& \left.+\left\{\frac{2 s+3}{1-s^{2}} \frac{\partial B}{\partial y^{i}}+\frac{\left(2 s^{2}+6 s+2\right) B}{\left(1-s^{2}\right)^{2}} s_{y^{i}}\right\}\left\langle\left[v, v_{j}\right]_{\mathfrak{k}}, v\right\rangle\right],
\end{aligned}
$$

where $v \in \mathfrak{k}$ corresponds to the 1 -form $\beta$ and $\mathfrak{k}$ is identified with the tangent space $T_{H}(G / H)$ of $G / H$ at the origin $H$.

Proof. From the equation (4.4), we can write $S$ - curvature at the origin as follows

$$
S(H, y)=\phi_{2}+\psi_{2},
$$

where

$$
\phi_{2}=\frac{B}{\alpha}\left\langle[v, y]_{\mathfrak{k}}, y\right\rangle \text { and } \psi_{2}=\frac{2 s+3}{1-s^{2}} B\left\langle[v, y]_{\mathfrak{k}}, v\right\rangle
$$

Therefore, mean Berwald curvature is

$$
E_{i j}=\frac{1}{2} \frac{\partial^{2} S}{\partial y^{i} \partial y^{j}}=\frac{1}{2}\left(\frac{\partial^{2} \phi_{2}}{\partial y^{i} \partial y^{j}}+\frac{\partial^{2} \psi_{2}}{\partial y^{i} \partial y^{j}}\right),
$$

where $\frac{\partial^{2} \phi_{2}}{\partial y^{i} \partial y^{j}}$ and $\frac{\partial^{2} \psi_{2}}{\partial y^{i} \partial y^{j}}$ are calculated as follows:

$$
\begin{aligned}
\frac{\partial \phi_{2}}{\partial y^{j}}= & \frac{\partial}{\partial y^{j}}\left(\frac{B}{\alpha}\left\langle[v, y]_{\mathfrak{k}}, y\right\rangle\right) \\
= & \left(\frac{1}{\alpha} \frac{\partial B}{\partial y^{j}}-\frac{B}{\alpha^{2}} \frac{y_{j}}{\alpha}\right)\left\langle[v, y]_{\mathfrak{k}}, y\right\rangle+\frac{B}{\alpha}\left(\left\langle\left[v, v_{j}\right]_{\mathfrak{k}}, y\right\rangle+\left\langle[v, y]_{\mathfrak{k}}, v_{j}\right\rangle\right) \\
\frac{\partial^{2} \phi_{2}}{\partial y^{i} \partial y^{j}}= & \frac{\partial}{\partial y^{i}}\left\{\left(\frac{1}{\alpha} \frac{\partial B}{\partial y^{j}}-\frac{B y_{j}}{\alpha^{3}}\right)\left\langle[v, y]_{\mathfrak{k}}, y\right\rangle+\frac{B}{\alpha}\left(\left\langle\left[v, v_{j}\right]_{\mathfrak{k}}, y\right\rangle+\left\langle[v, y]_{\mathfrak{k}}, v_{j}\right\rangle\right)\right\} \\
= & \left(\frac{1}{\alpha} \frac{\partial^{2} B}{\partial y^{i} \partial y^{j}}-\frac{y_{i}}{\alpha^{3}} \frac{\partial B}{\partial y^{j}}-\frac{y_{j}}{\alpha^{3}} \frac{\partial B}{\partial y^{i}}-\frac{B}{\alpha^{3}} \delta_{i}^{j}+\frac{3 B}{\alpha^{5}} y_{i} y_{j}\right)\left\langle[v, y]_{\mathfrak{k}}, y\right\rangle \\
& +\left(\frac{1}{\alpha} \frac{\partial B}{\partial y^{j}}-\frac{B}{\alpha^{3}} y_{j}\right)\left(\left\langle\left[v, v_{i}\right]_{\mathfrak{k}}, y\right\rangle+\left\langle[v, y]_{\mathfrak{k}}, v_{i}\right\rangle\right) \\
& +\left(\frac{1}{\alpha} \frac{\partial B}{\partial y^{i}}-\frac{B}{\alpha^{3}} y_{i}\right)\left(\left\langle\left[v, v_{j}\right]_{\mathfrak{k}}, y\right\rangle+\left\langle[v, y]_{\mathfrak{k}}, v_{j}\right\rangle\right) \\
& +\frac{B}{\alpha}\left(\left\langle\left[v, v_{j}\right]_{\mathfrak{k}}, v_{i}\right\rangle+\left\langle\left[v, v_{i}\right]_{\mathfrak{k}}, v_{j}\right\rangle\right),
\end{aligned}
$$


and

$$
\begin{aligned}
& \frac{\partial \psi_{2}}{\partial y^{j}}=\frac{\partial}{\partial y^{j}}\left(\frac{(2 s+3) B}{1-s^{2}}\left\langle[v, y]_{\mathfrak{k}}, v\right\rangle\right) \\
& =\left\{\frac{2 s+3}{1-s^{2}} \frac{\partial B}{\partial y^{j}}+\frac{\left(2 s^{2}+6 s+2\right) B}{\left(1-s^{2}\right)^{2}} s_{y^{j}}\right\}\left\langle[v, y]_{\mathfrak{k}}, v\right\rangle+\frac{(2 s+3) B}{1-s^{2}}\left\langle\left[v, v_{j}\right]_{\mathfrak{k}}, v\right\rangle \text {, } \\
& \frac{\partial^{2} \psi_{2}}{\partial y^{i} \partial y^{j}}=\frac{\partial}{\partial y^{i}}\left[\left\{\frac{2 s+3}{1-s^{2}} \frac{\partial B}{\partial y^{j}}+\frac{\left(2 s^{2}+6 s+2\right) B}{\left(1-s^{2}\right)^{2}} s_{y^{j}}\right\}\left\langle[v, y]_{\mathfrak{k}}, v\right\rangle\right. \\
& \left.+\frac{(2 s+3) B}{1-s^{2}}\left\langle\left[v, v_{j}\right]_{\mathfrak{k}}, v\right\rangle\right] \\
& =\left\{\frac{2 s+3}{1-s^{2}} \frac{\partial^{2} B}{\partial y^{i} \partial y^{j}}+\frac{2 s^{2}+6 s+2}{\left(1-s^{2}\right)^{2}} s_{y^{i}} \frac{\partial B}{\partial y^{j}}+\frac{2 s^{2}+6 s+2}{\left(1-s^{2}\right)^{2}} s_{y^{j}} \frac{\partial B}{\partial y^{i}}\right. \\
& \left.+\frac{\left(4 s^{3}+18 s^{2}+12 s+6\right) B}{\left(1-s^{2}\right)^{3}} s_{y^{i}} s_{y^{j}}+\frac{\left(2 s^{2}+6 s+2\right) B}{\left(1-s^{2}\right)^{2}} s_{y^{i} y^{j}}\right\}\left\langle[v, y]_{\mathfrak{k}}, v\right\rangle \\
& +\left\{\frac{2 s+3}{1-s^{2}} \frac{\partial B}{\partial y^{j}}+\frac{\left(2 s^{2}+6 s+2\right) B}{\left(1-s^{2}\right)^{2}} s_{y^{j}}\right\}\left\langle\left[v, v_{i}\right]_{\mathfrak{k}}, v\right\rangle \\
& +\left\{\frac{2 s+3}{1-s^{2}} \frac{\partial B}{\partial y^{i}}+\frac{\left(2 s^{2}+6 s+2\right) B}{\left(1-s^{2}\right)^{2}} s_{y^{i}}\right\}\left\langle\left[v, v_{j}\right]_{\mathfrak{k}}, v\right\rangle \text {. }
\end{aligned}
$$

Substituting all above values in the equation (5.2), we get the formula (5.1).

\section{RE F E R E N C E S}

1. S. I. Amari and H. NAGAOKA: Methods of Information Geometry. Translations of Mathematical Monographs, AMS, 191, Oxford Univ. Press, 2000.

2. P. L. Antonelli, R. S. Ingarden and M. Matsumoto: The Theory of Sprays and Finsler spaces with Applications in Physics and Biology. Vol. 58, Springer Science \& Business Media, 2013.

3. D. BAo, S. S. Chern and Z. Shen: An Introduction to Riemann-Finsler Geometry. Springer-Verlag, New York, 2000.

4. L. BERWALD: $\ddot{U}$ ber dien-dimensionalen Geometrien konstanter Krümmung, in denen die Geraden die kürzesten sind. Mathematische Zeitschrift. 30 (1) (1929), 449-469.

5. X. Cheng And Z. Shen: A class of Finsler metrics with isotropic S-curvature. Israel Journal of Mathematics. 169 (2009), 317-340.

6. S. S. CHERN: Finsler geometry is just Riemannian geometry without quadratic restriction. Notices of AMS. 43 (9) (1996), 959-963.

7. S. S. Chern and Z. Shen: Riemann-Finsler Geometry. Nankai Tracts in Mathematics, Vol. 6, World Scientific, Singapore, 2005.

8. S. Deng: The S-curvature of homogeneous Randers spaces. Differential Geometry and its Applications. 27 (2009), 75-84. 
9. S. DenG: Homogeneous Finsler Spaces. Springer Monographs in Mathematics, New York, 2012.

10. S. Deng and Z. Hou: The group of isometries of a Finsler space. Pacific J. Math. 207 (2002), 149-155.

11. S. Deng and Z. Hou: Invariant Randers metrics on Homogeneous Riemannian manifolds. Journal of Physics A: Mathematical and General. 37 (2004), 4353-4360; Corrigendum, ibid, 39 (2006), 5249-5250.

12. S. Deng and X. WANG: The S-curvature of homogeneous $(\alpha, \beta)$-metrics. Balkan Journal of Geometry and Its Applications. 15 (2) (2010), 39-48.

13. R. GARDNER and G. Wilkens: A pseudo-group isomorphism between control systems and certain generalized Finsler structures. Contemporary Mathematics. 196 (1996), 231-244.

14. M. HAShiguChI and Y. ICHIJyō: Randers spaces with rectilinear geodesics. Rep. Fac. Sci., Kagoshima Univ., (Math., Phys. \& Chem.). 13 (1980), 33-40.

15. Z. Hu and S. Deng: Homogeneous Randers spaces with isotropic S-curvature and positive flag curvature. Mathematische Zeitschrift. 270 (2012), 989-1009.

16. G. Kron: Non-Riemannian dynamics of rotating electrical machinery. Studies in Applied Mathematics. 13 (1934), 103-194.

17. M. мatsumoto: On C-reducible Finsler-spaces. Tensor, N. S. 24 (1972), 29-37.

18. M. Matsumoto: On Finsler spaces with Randers metric and special forms of important tensors. Journal of mathematics of Kyoto University. 14 (3) (1974), 477-498.

19. K. Nomizu: Invariant affine connections on homogeneous spaces. Amer. J. Math. 76 (1954), 33-65.

20. G. RANDERS: On an asymmetric metric in the four-space of general relativity. Phys. Rev. 59 (1941), 195-199.

21. G. SHANKER and K. KAUR: Naturally reductive homogeneous space with an invariant $(\alpha, \beta)$-metric. Lobachevskii Journal of Mathematics. 40 (2) (2019), 210-218.

22. G. Shanker and K. KaUR: Homogeneous Finsler space with infinite series $(\alpha, \beta)$ metric. Applied Sciences. 21 (2019), 220-236.

23. G. Shanker and S. RANi: On S-Curvature of a Homogeneous Finsler space with square metric. International Journal of Geometric Methods in Modern Physics. 17 (02) (2020), 2050019 (16 pages).

24. G. Shanker, S. RANi and K. KAUR: Dually flat Finsler spaces associated with Randers- $\beta$ change. Journal of Rajasthan Academy of Physical Sciences. 18 (1-2) (2019), 95-106.

25. Z. Shen: Volume comparison and its applications in Riemann-Finsler geometry. Advances in Mathematics. 128 (1997), 306-328.

26. Z. SHen: Finsler metrics with $K=0$ and $S=0$. Canadian Journal of Mathematics. 55 (2003), 112-132.

27. Z. SHEN: Non-positively curved Finsler manifolds with constant S-curvature. Mathematische Zeitschrift. 249 (2005), 625-639.

28. Z. Shen and C. YU: On Einstein square metrics. Publicationes Mathematicae Debrecen. 85 (3-4) (2014), 413-424.

29. Q. XIA: On a class of projectively flat Finsler metrics. Differential Geometry and its Applications, 44 (2016), 1-16. 
30. M. Xu and S. Deng: Homogeneous $(\alpha, \beta)$-spaces with positive flag curvature and vanishing $S$-curvature,. Nonlinear Analysis. 127 (2015), 45-54.

31. C. YU and H. ZHU: Projectively flat general $(\alpha, \beta)$-metrics with constant flag curvature. Journal of Mathematical Analysis and Applications. 429 (2) (2015), 1222-1239.

\section{Sarita Rani}

Department of Mathematics and Statistics

Central University of Punjab, Bathinda

151001 Punjab, India

saritas.ss92@gmail.com

Gauree Shanker

Department of Mathematics and Statistics

Central University of Punjab, Bathinda

151001 Punjab, India

grshnkr2007@gmail .com 